\title{
Biotimer assay: A reliable and rapid method for the evaluation of central venous catheter microbial colonization
}

\author{
Luigi Rosa, Antimo Cutone, Monica Coletti, Maria Stefania Lepanto, Mellani Scotti, Piera Valenti, \\ Giammarco Raponi, Maria Cristina Ghezzi ${ }^{1}$, Francesca Berlutti \\ Department of Public Health and Infectious Diseases, Sapienza University of Rome, P.ale A. Moro, 5, 00185 Rome, Italy
}

\section{A R T I C L E I N F O}

\section{Keywords:}

BioTimer assay

Central venous catheter colonization

Biofilm

Catheter-related bloodstream infection

\begin{abstract}
A B S T R A C T
Adherent bacteria and biofilm frequently colonize central venous catheters (CVCs). CVC colonization is correlated to infections and particularly to bloodstream ones. The classical microbiological methods to determine of CVC colonization are not fully reliable and are time-consuming. BioTimer Assay (BTA) is a biological method already used to count bacteria adherent to abiotic surfaces and biofilm without sample manipulation. BTA employs specific reagents whose color changed according to bacterial metabolism. BTA is based on the principle that a metabolic reaction will be faster when more bacteria are present in the sample. Therefore, the time required for color changes of BTA reagents determines the number of bacteria present in the sample through a correlation line. Here, for the first time, we applied BTA and a specifically developed laboratory procedure to evaluate CVC colonization in comparison with the routine microbiological method (RMM). 125 CVCs removed from patients for suspected catheter-related bloodstream infection (CRBSI) or at hospital discharge were examined. BTA was reliable in assessing sterility and CVC colonization (100\% agreement with RMM) and in recognizing the presence of fermenting or non-fermenting bacteria ( $97.1 \%$ agreement with RMM) shortening the analytical time by between 2- and 3-fold. Moreover, the reliability of BTA as early alert of CRBSI was evaluated. The sensitivity, specificity, positive, and negative predictive values for BTA as an early alert of CRBSI were 100, 40.0, 88.8 and $100 \%$, respectively.

In conclusion, BTA and the related laboratory procedure should be incorporated into routine microbiological methods since it can be considered a reliable tool to evaluate CVC colonization in a very short time and a rapid alert for CRBSIs.
\end{abstract}

\section{Introduction}

Central venous catheters (CVCs) are a mainstay for management of critically ill patients. However, CVCs may be colonized on intra- and extra-luminal surfaces by microorganisms in adherent and biofilm lifestyle (Dobbins et al., 2003; Gominet et al., 2017). CVC colonization is correlated to infectious episodes and, in particular, the catheter-related bloodstream infections (CRBSIs) are characterized by high rates of morbidity and mortality (Pratt et al., 2001; Chopra et al., 2013; Gahlot et al., 2014; Yousif et al., 2015). Moreover, the biofilms colonizing CVCs show antimicrobial resistance significantly higher than the planktonic counterparts (Donlan and Costerton, 2002; Hall-Stoodley et al., 2004; Pantanella et al., 2008) prolonging the length of hospitalization and increasing healthcare costs (Kaye et al., 2014; Gahlot et al., 2014).

The microbiological methods for the routine analysis of CVC colonization are semi-quantitative and actually based on the roll-plate technique (Maki et al., 1977; Mermel et al., 2009) or on the detachment of adherent microbes by vortex or sonication and the count of the detached microorganisms by the colony forming unit (CFU) technique (Cleri et al., 1980; Sherertz et al., 1990; Wengrovitz et al., 1991). Unfortunately, no one of these methods is fully reliable. Indeed, the rollplate technique does not determine the intra-luminal colonization of CVCs (Dobbins et al., 2003; Mermel et al., 2009; Guembe et al., 2016; Gominet et al., 2017) and the vortex or sonication methods do not ensure the detachment of all microbes (Pantanella et al., 2013; Freitas

\footnotetext{
Abbreviations: BTA, BioTimer Assay; CVCs, central venous catheters; RMM, routine microbiological method; CRBSI, catheter-related bloodstream infection

* Corresponding author.

E-mail addresses: luigi.rosa@uniroma1.it (L. Rosa), antimo.cutone@uniroma1.it (A. Cutone), monica.coletti@uniroma1.it (M. Coletti),

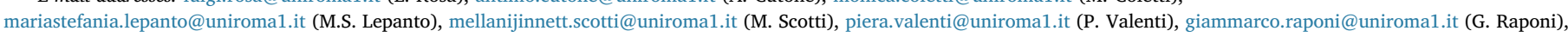
francesca.berlutti@uniroma1.it (F. Berlutti).

${ }^{1}$ This manuscript is dedicated to Maria Cristina Ghezzi who prematurely passed away.
} 
et al., 2014). It is evident that a rapid and reliable microbiological method for the early determination of CVC colonization is extremely important. BioTimer Assay (BTA) is a biological method that counts adherent bacteria and biofilm without sample manipulation as vortex or sonication to detach biomass and cultivation, thus overcoming the above-mentioned limits (Berlutti et al., 2003; Pantanella et al., 2008; Pantanella et al., 2011; Berlutti et al., 2014; Srivastava and Bhargava, 2016). In particular, BTA is based on the principle that a metabolic reaction will be faster when more bacteria are present in the sample. BTA employs specific reagents whose color changes according to bacterial metabolism. The time required for color changes of BTA reagents is inversely related to initial bacterial concentration. Therefore, the time for color change determines the number of bacteria present in the sample at Time 0 through genus-specific correlation lines. In addition, BTA was successfully employed to evaluate biofilm colonization of medical devices (Hess et al., 2011; Wells et al., 2011; Romeo et al., 2015).

Here, for the first time, BTA was applied to evaluate microbial colonization of CVCs removed from patients hospitalized at the University Hospital Policlinico Umberto I, Rome, Italy. For this purpose, a specific laboratory procedure was developed. The performance of BTA and the related laboratory procedure was compared with the method of Cleri et al. (1980) routinely employed at the Clinical Microbiological Laboratory of the same hospital. Furthermore, the correlation between BTA results and CVC-related bloodstream infection (CRBSI) diagnosis was investigated. The data presented strongly indicate that BTA can be usefully employed to evaluate CVC colonization and can represent an alert tool for CRBSI.

\section{Materials and methods}

\subsection{Bacterial strains and culture media}

Staphylococcus epidermidis ATCC 12228 and Pseudomonas aeruginosa ATCC 15692 (PAO1) were streaked on 5\% Columbia blood agar (CBA) plates (Oxoid LTD, England) before the experiments to check purity.

\subsection{BioTimer Assay (BTA)}

BioTimer Assay (BTA) allows counting bacteria in planktonic, adherent and biofilm lifestyle (Berlutti et al., 2003; Pantanella et al., 2008; Pantanella et al., 2011; De Giusti et al., 2011; Berlutti et al., 2014). BTA employs two specific reagents: BioTimer-phenol red (BTPR) and BioTimer-resazurin (BT-RZ) whose color changes due to microbial metabolism (Berlutti et al., 2003; Pantanella et al., 2008; Pantanella et al., 2011). In particular, BT-PR reagent changed red-toyellow due to reagent acidification when inoculated with fermenting bacteria and BT-RZ reagent changed blue-to-pink due to redox reaction when inoculated with fermenting or non-fermenting bacteria (Pantanella et al., 2011). BT-PR and BT-RZ reagents were prepared as previously described with slight modification (Pantanella et al., 2008; Pantanella et al., 2011; Berlutti et al., 2014). Briefly, to prepare BR-PR reagent, $3.7 \mathrm{~g}$ of Brain Heart Infusion (BHI; Oxoid Ltd., UK) were dissolved in $940 \mathrm{ml}$ of distilled water. After sterilization at $115{ }^{\circ} \mathrm{C}$ for $15 \mathrm{~min}, 50 \mathrm{ml}$ of $10 \%$ filtered glucose solution and $10 \mathrm{ml}$ of filtered $0.25 \%$ phenol red (Sigma Aldrich, Italy) were added. If necessary, the $\mathrm{pH}$ was adjusted to $7.2 \pm 0.1$. The final BT-PR reagent appeared clear and red. To prepare the BT-RZ reagent, $10 \mathrm{ml}$ of freshly prepared and filtered $0.1 \%$ aqueous resazurin solution (Sigma-Aldrich, Italy) was added to $990 \mathrm{ml}$ sterile BHI broth prepared as above described (Oxoid). If necessary, the $\mathrm{pH}$ was adjusted to $\mathrm{pH} 7.0 \pm 0.1$. The final BT-RZ reagent appeared clear and blue.

The time required for color change of BTA reagents is correlated to initial bacterial concentration by correlation lines. To draw the correlation lines, serial two-fold dilutions of planktonic overnight broth cultures of $S$. epidermidis and of $P$. aeruginosa in $1 \mathrm{ml}$ of BT-PR and BT-

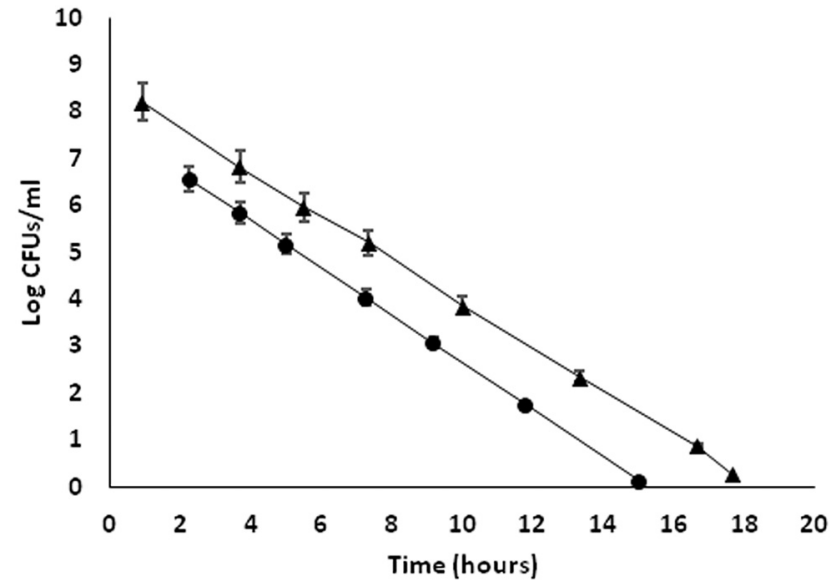

Fig. 1. BioTimer Assay correlation lines.

Legend: Correlation lines of $S$. epidermidis ATCC 12228 (circles) and of $P$. aeruginosa ATCC 15692 (triangles) in BT-PR and BT-RZ reagents, respectively.

$\mathrm{RZ}$ reagents, respectively, were performed in 24-well plates (BD, Italy), and simultaneously counted using the colony forming unit (CFU) method. Briefly, to carry out the CFU method, bacterial suspensions were diluted in sterile saline solution and plated on BHI plates. After $24 \mathrm{~h}$ of incubation, colonies were counted. The number of CFUs in the initial bacterial suspensions was determined by multiplying the number of counted CFUs on BHI plates with the dilution factor. The time (in hours) required for color changing of the inoculated BT-PR and BT-RZ reagents was recorded and plotted versus the corresponding $\mathrm{CFU}$ values (Fig. 1). The equations and the linear correlation coefficients describing the correlation lines were calculated for each microorganism on the whole data set and were: $\mathrm{y}=-0.5045 \mathrm{x}+7.6959 \mathrm{R}^{2}=0.9891$ for $S$. epidermidis and $\mathrm{y}=-0.4675 \mathrm{x}+8.5421$ and $\mathrm{r}^{2}=0.9968$ for $P$. aeruginosa. As the correlation lines correlated the time for the color change of BTA reagents with the number of planktonic CFUs, the number of bacteria in biofilm was expressed as planktonic-equivalent CFUs (PECFUs) (Pantanella et al., 2008).

\subsection{Experimental colonization of central venous catheters}

Sterile CVCs (Multi-Lumen Central Venous Catheterization Set, Arrow Italy) were aseptically cut in $1 \mathrm{~cm}$-segments and immersed in sterile saline $(0.9 \% \mathrm{NaCl})$ supplemented with $2 \%$ human serum pooled from healthy volunteers. After $30 \mathrm{~min}$ incubation, the CVC pieces were washed three times with sterile saline and transferred into sterile 24well flat-bottom plates. A total of $1 \mathrm{ml}$ of BHI broth containing about $10^{4}$ CFUs of planktonic log phase cultures of $S$. epidermidis ATCC 12228 or $P$. aeruginosa ATCC 15692 (PAO1) were added to each well. CVCs were incubated for 2 and $24 \mathrm{~h}$ to have adherent bacteria and biofilm, respectively. After incubation, bacteria were counted using the culture method according to Cleri et al. (1980) with slight modification. Briefly, the CVC segments were transferred into sterile tubes containing $1 \mathrm{ml}$ of BHI broth and then vortexed for $30 \mathrm{~s}$ to detach bacteria. Detached bacteria were counted by CFU method on BHI agar. To evaluate the residual adherent bacteria after vortex, the CVC segments were immersed in $1 \mathrm{ml}$ of BT-RP and BT-RZ reagents and counted by BTA.

\subsection{Central venous catheters collection}

CVCs were randomly selected among those received at the Clinical Microbiology laboratory at "Azienda Policlinico Umberto I", Hospital of Sapienza University of Rome, Italy during a two years period (March 2014 to March 2016). CVCs were removed from hospitalized patients either at the end of the clinical therapy or for the clinical suspicion of CRBSI (Mermel et al., 2009). 


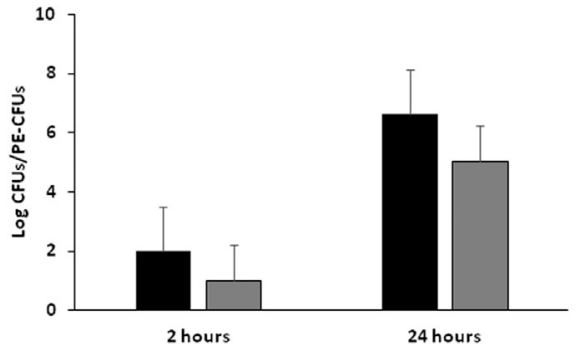

A

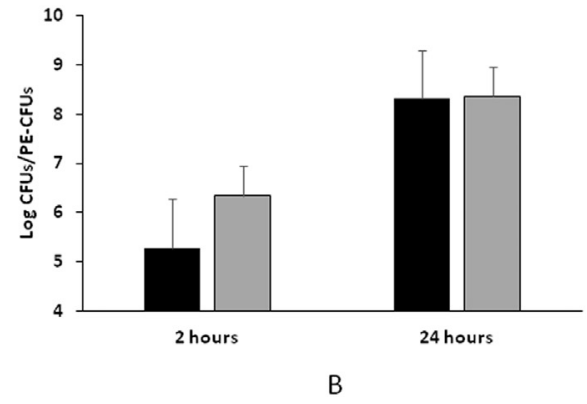

Fig. 2. Counts of Staphylococcus epidermidis ATCC 12228 and Pseudomonas aeruginosa ATCC 15692 adherent on experimentally colonized central venous catheters.

Legend: colonized central venous catheters were vortexed for $30 \mathrm{~s}$ and detached bacteria were counted and expressed as CFUs (black columns); the residual adherent bacteria after vortex were counted using BioTimer Assay and expressed as planktonic-equivalent CFUs (PE-CFUs) (grey columns) (for details see M \& M section); panel A: $S$. epidermidis; panel B: $P$. aeruginosa.

\subsection{Laboratory procedures}

\subsubsection{Routine microbiological method (RMM)}

CVCs were examined at the Clinical Microbiology Laboratory by RMM according to Cleri et al. (1980) with slight modification. In brief, approximately $5 \mathrm{~cm}$ of the tip distal part of the CVCs was placed in $1 \mathrm{ml}$ of BHI broth and vigorously shaken on a vortex for $30 \mathrm{~s}$. A volume of $10 \mu \mathrm{l}$ of the suspension was then streaked on $5 \%$ Columbia Blood Agar (CBA) plates (Oxoid Ltd., UK). After incubation at $37{ }^{\circ} \mathrm{C}$ for $24 \mathrm{~h}$, the number of CFUs was determined. The CBA negative plates were incubated for further $24 \mathrm{~h}$ to assess the sterility of the culture. The identification of the isolates was performed using the Vitek $2^{\circledR}$ automated method (Biomerieux Diagnostic System, Inc., St. Louis, MI). CVC colonization was defined when $\geq 10^{2} \mathrm{CFUs} / \mathrm{ml}$ of $\mathrm{S}$. epidermidis or coagulase-negative Staphylococcus (CoNS) were counted or when microbes other than S. epidermidis or CoNS were isolated irrespective of the bacterial count.

\subsubsection{BTA laboratory procedure}

After the vortex treatment (see above), the $5 \mathrm{~cm}$-tip distal part of the CVCs were sent to Microbiology Laboratory at Department of Public Health and Infectious Diseases, Sapienza University of Rome, Italy to be examined by BTA and related procedure. The CVCs were cut in two identical pieces of $2.5 \mathrm{~cm}$ and completely immersed in $5 \mathrm{ml}$ of BT-PR and BT-RZ reagents. The BTA reagents were incubated at $37{ }^{\circ} \mathrm{C}$ without agitation and the color was monitored every 30 min during the first $8 \mathrm{~h}$ and then every hour for a total of $24 \mathrm{~h}$. The time required for color changing was recorded and used to evaluate the number of PE-CFUs. In particular, the changing of BT-PR alone or of both BT-PR and BT-RZ reagents indicated the presence of fermenting microbe(s) in pure or mixed flora. In this case, the $S$. epidermidis correlation line was used to calculate the PE-CFUs. The changing of the sole BT-RZ reagent indicated the presence of non-fermenting microbe(s) and hence the correlation line of $P$. aeruginosa was used to evaluate the PE-CFUs. The BTPR and BT-RZ inoculated reagents, whose color did not change within the first $24 \mathrm{~h}$ of incubation, were considered sterile.

\subsection{Statistical analysis}

Mean values and standard deviations were calculated on whole data sets obtained from at least three independent experiments. Correlation lines were obtained by linear regression analysis, and linear correlation coefficients were calculated from the equation: $r=(n \Sigma x y-\Sigma x \Sigma y) /$ $(\operatorname{sqrt}((\mathrm{n} \Sigma \mathrm{x} 2-(\Sigma \mathrm{x}) 2)(\mathrm{n} \Sigma \mathrm{y} 2-(\Sigma \mathrm{y}) 2)))$. The sensitivity, specificity, positive predictive (PPV), and negative predictive values (NPV) for BTA as a rapid alert of CRBSI were evaluated.

\subsection{Ethics}

Written patient consents were not required because of the observational nature of this study. The CVCs were removed by the healthcare professionals at the hospitalization site of the patients and sent to the laboratory for analysis, and therefore the authors did not collect clinical samples. Patient data concerning microbiological analysis of CVC and blood samples were collected from an electronic database. Laboratory staff members not involved in the study anonymized these records.

\section{Results}

\subsection{Experimental colonization of CVCs}

Experimental colonization of CVCs was performed to validate BTA in counting adherent bacteria to CVCs. CVC colonization was evaluated counting both the vortex-detached bacteria by CFU method and the residual adherent bacteria after vortexing by BTA. Other achieved aims were to determine if the vortex procedure did not completely detach $S$. epidermidis and P. aeruginosa (Fig. 2, black columns) and that BTA was able to count the residual adherent $S$. epidermidis and $P$. aeruginosa (Fig. 2, grey columns).

Therefore, BTA was judged reliable and it was employed in analyzing CVCs removed from the hospitalized patients.

\subsection{Evaluation of bacterial colonization of CVCs}

During the two-year period, 125 CVCs were analyzed of which 29 were removed for suspicion of CRBSI and 96 at the hospital discharge. Out the CVCs, 90 were considered sterile using RMM since no microbial growth was observed on CBA plates after $48 \mathrm{~h}$ of incubation. These CVCs were considered sterile also for BTA, as they not induced the color change of BTA reagents within $24 \mathrm{~h}$ of incubation $(100 \%$ agreement with RMM).

Out of the 35 non-sterile CVCs, 29 were all those removed for suspicion of CRBSI and six were removed at hospital discharge (Table 1).

According to RMM (Table 1), 27 CVCs gave rise to the development of microbial colonies after $24 \mathrm{~h}$ of incubation, and eight after $48 \mathrm{~h}$. In 30 cases, the counts were higher than $10^{2} \mathrm{CFUs} / \mathrm{ml}$ and the CVCs were considered colonized. In five cases counts lower than $10^{2} \mathrm{CFUs} / \mathrm{ml}$ were determined. Of those CVCs, two were considered colonized since Alcaligenes spp., Serratia spp. and Corynebacterium spp. were isolated and three were considered as non-colonized since CoNS or S. epidermidis were identified. Totally, RMM recognized as colonized 32 CVCs.

Using BTA (Table 1), all 35 CVCs determined the color change of one or both BTA reagents within $24 \mathrm{~h}$ of incubation indicating the presence of adherent microbes (100\% agreement with RMM). The bacterial counts ranged from $7.6 * 10^{6}$ to $\leq 10^{2}$ PE-CFUs. In particular, 30 CVCs showed counts higher and five lower than $10^{2}$ PE-CFUs. Applying the criteria of the RMM, BTA correctly recognized as colonized 32 CVCs (100\% agreement).

In addition to bacterial count, BTA method allowed evaluating the presence of fermenting and non-fermenting microbes through the observation of the color change of BTA reagents (Table 1). Non-fermenting bacteria changed BT-RZ reagent and fermenting microorganisms in pure or in mixed flora with non-fermenting bacteria changed both BT-PR and BT-RZ reagents. In particular, seven CVCs changed BT$\mathrm{RZ}$ reagent only indicating the presence of non-fermenting bacteria, 
Table 1

Performance of BioTimer Assay in comparison to the routine microbiological method in evaluating CVC colonization.

\begin{tabular}{|c|c|c|c|c|c|c|}
\hline \multicolumn{2}{|l|}{$\mathrm{CVC}^{\mathrm{a}}$} & \multicolumn{3}{|l|}{ BioTimer Assay } & \multicolumn{2}{|c|}{ Routine microbiological method } \\
\hline Acronym & Removed for & $\begin{array}{l}\text { Changing time }{ }^{\mathrm{b}} \\
\text { (hours) }\end{array}$ & PE-CFUs/CVC & $\mathrm{F} / \mathrm{NF}^{\mathrm{d}}$ & Colonization $^{\mathrm{e}}$ & Microbe identification ${ }^{\mathrm{f}}$ \\
\hline $\mathrm{DE}$ & S-CRBSI & 3 & $7.6 * 10^{6}$ & F & $\mathrm{Y}$ & Pseudomonas spp., Enterococcus faecalis, Proteus mirabilis, CoNS \\
\hline CG & S-CRBSI & 3.5 & $3.2 * 10^{7}$ & $\mathrm{NF}$ & $\mathrm{Y}$ & Acinetobacter baumanni, Serratia marcescens \\
\hline DAE & S-CRBSI & 4 & $2.4 * 10^{6}$ & $\mathrm{~F}$ & $\mathrm{Y}$ & Staphylococcus aureus, A. baumanni, P. vulgaris \\
\hline LR & S-CRBSI & 5 & $7.4 * 10^{5}$ & $\mathrm{~F}$ & $\mathrm{Y}$ & Klebsiella pneumoniae \\
\hline MM & S-CRBSI & 5 & $7.4 * 10^{5}$ & $\mathrm{~F}$ & $\mathrm{Y}$ & E. faecium \\
\hline FD & S-CRBSI & 5.5 & $4.2 * 10^{5}$ & $\mathrm{~F}$ & $\mathrm{Y}$ & Klebsiella spp., Candida spp. \\
\hline $\mathrm{CA}$ & S-CRBSI & 6 & $2.3 * 10^{5}$ & $\mathrm{~F}$ & $\mathrm{Y}$ & Enterococcus spp., Klebsiella spp., Pseudomonas spp. \\
\hline PM & S-CRBSI & 6 & $2.2 * 10^{6}$ & NF & $\mathrm{Y}$ & P.aeruginosa \\
\hline ML & S-CRBSI & 6.5 & $1.3 * 10^{5}$ & $\mathrm{~F}$ & $\mathrm{Y}$ & A. baumanni, Pseudomonas spp., Escherichia coli \\
\hline ZA & S-CRBSI & 6.5 & $1.3 * 10^{5}$ & $\mathrm{~F}$ & $\mathrm{Y}$ & S. aureus \\
\hline $\mathrm{CP}$ & S-CRBSI & 7 & $7.5 * 10^{5}$ & NF & $\mathrm{Y}$ & A. baumanni, Pseudomonas spp. \\
\hline MAL & S-CRBSI & 7 & $7.5 * 10^{5}$ & NF & $\mathrm{Y}$ & A. baumanni, Pseudomonas spp. \\
\hline MA & S-CRBSI & 7 & $7.3 * 10^{4}$ & $\mathrm{~F}$ & $\mathrm{Y}$ & S. aureus \\
\hline BV & S-CRBSI & 8 & $2.5 * 10^{5}$ & NF & $\mathrm{Y}$ & A. baumanni \\
\hline CR & S-CRBSI & 8 & $2.3 * 10^{4}$ & $\mathrm{~F}$ & $\mathrm{Y}$ & CoNS \\
\hline PAM & S-CRBSI & 8 & $2.3 * 10^{4}$ & $\mathrm{~F}$ & $\mathrm{Y}$ & Candida spp. \\
\hline DO & S-CRBSI & 8 & $2.3 * 10^{4}$ & $\mathrm{~F}$ & $\mathrm{Y}$ & C. albicans \\
\hline MIR & S-CRBSI & 8 & $2.3 * 10^{4}$ & $\mathrm{~F}$ & $\mathrm{Y}$ & E. coli \\
\hline $\mathrm{CM}$ & S-CRBSI & 11 & $7.0 * 10^{2}$ & $\mathrm{~F}$ & $\mathrm{Y}$ & CoNS \\
\hline LD & S-CRBSI & 11 & $7.0 * 10^{2}$ & $\mathrm{~F}$ & $\mathrm{Y}$ & CoNS \\
\hline AG & S-CRBSI & 11 & $7.0 * 10^{2}$ & $\mathrm{~F}$ & $\mathrm{Y}$ & CoNS \\
\hline RA & S-CRBSI & 11 & $7.0 * 10^{2}$ & $\mathrm{~F}$ & $\mathrm{Y}$ & S. epidermidis \\
\hline CAG & $\mathrm{HD}$ & 11 & $7.0 * 10^{2}$ & $\mathrm{~F}$ & $\mathrm{Y}$ & S. epidermidis \\
\hline BA & S-CRBSI & 12 & $2.2 * 10^{2}$ & $\mathrm{~F}$ & $\mathrm{Y}$ & CoNS \\
\hline $\mathrm{AD}$ & S-CRBSI & 12 & $2.2 * 10^{2}$ & $\mathrm{~F}$ & $\mathrm{Y}$ & K. ornitholytica, CoNS \\
\hline GV & S-CRBSI & 12 & $2.2 * 10^{2}$ & $\mathrm{~F}$ & $\mathrm{Y}$ & C. albicans \\
\hline CEC & S-CRBSI & 12 & $2.2 * 10^{2}$ & $\mathrm{~F}$ & $\mathrm{Y}$ & CoNS \\
\hline MOR & S-CRBSI & 12 & $3.4 * 10^{3}$ & NF & $\mathrm{Y}$ & A. baumanni \\
\hline PA & $\mathrm{HD}$ & 12 & $2.2 * 10^{2}$ & $\mathrm{~F}$ & $\mathrm{Y}$ & Candida spp. \\
\hline PRO & S-CRBSI & 13 & $1.2 * 10^{3}$ & NF & $\mathrm{Y}$ & A. baumanni, Pseudomonas spp. \\
\hline RM2 & $\mathrm{HD}$ & 13 & $<10^{2}$ & $\mathrm{~F}$ & $\mathrm{~N}$ & CoNS \\
\hline DIE & HD & 15 & $<10^{2}$ & $\mathrm{~F}$ & $\mathrm{~N}$ & S. epidermidis \\
\hline DE2 & HD & 15 & $<10^{2}$ & $\mathrm{~F}$ & $\mathrm{~N}$ & CoNS \\
\hline $\mathrm{RM}$ & S-CRBSI & 18 & $<10^{2}$ & $\mathrm{~F}$ & $\mathrm{Y}$ & Alcaligenes spp., Serratia spp. \\
\hline GON & $\mathrm{HD}$ & 18 & $<10^{2}$ & $\mathrm{~F}$ & $\mathrm{Y}$ & Corynebacterium spp. \\
\hline
\end{tabular}

${ }^{a}$ CVC: central venous catheters were removed for suspected catheter-related bloodstream infection (S-CRBSI) or at hospital discharge (HD).

b Changing time of BioTimer Assay reagent(s).

${ }^{\mathrm{c}}$ Microbial counts were calculated using S. epidermidis and P. aeruginosa correlation lines and expressed as PE-CFUs (see 2.2 section for details).

${ }^{\mathrm{d}}$ F: changing of BT-PR reagent indicating the presence of fermenting microbe(s); NF: changing of BT-RZ reagent only indicating the presence of non-fermenting microbe(s).

e Y, colonized CVC; N: non-colonized CVC.

${ }^{\mathrm{f}}$ Staphylococcus identification was done at level species for $S$. aureus and S. epidermidis only. Staphylococci other than S. aureus and S. epidermidis are indicated as coagulase-negative staphylococci (CoNS).

while 28 CVCs determined the change of both BTA reagents indicating the presence of fermenting microbes (Table 1). Interestingly, Candida spp. also changed BTA reagent like to fermenting bacteria do. The presumptive identification of fermenting or non-fermenting microorganisms was confirmed in 34 out 35 cases (Table 1) $(97.1 \%$ agreement with RMM). One discrepancy was observed for CG CVC. In this case, BTA indicated the presence of non-fermenting bacteria while it was actually colonized by non-fermenting Acinetobacter spp. and fermenting Serratia spp.

\subsection{Time required for the evaluation of sterility and colonization of CVCs}

The time necessary for the evaluation of sterility and colonization of CVCs using the RMM and BTA was compared (Table 2).

The time required to analyze all CVCs was shorter using BTA than RMM (63\% of reduction). In particular, the time needed for the determination of sterility was reduced by $50 \%$ and that for the evaluation of CVC colonization to about $67 \%$.

\subsection{BTA as early alert of CRBSI}

Finally, we evaluated the reliability of BTA as an early alert of
Table 2

Time required for the evaluation of central venous catheter sterility and colonization using BioTimer Assay and routine microbiological method.

\begin{tabular}{llll}
\hline CVCs $^{\mathrm{a}}$ & No. & Hours & \\
\cline { 3 - 4 } & & BioTimer Assay & Routine microbiological method \\
\hline Sterile & 90 & 2160 & 4320 \\
Nonsterile & 35 & 309 & 1032 \\
Total & 125 & 2469 & 5352 \\
\hline
\end{tabular}

${ }^{\text {a }}$ CVCs: central venous catheters.

CRBSI. Therefore, the microbiological data of the 29 CVCs removed for suspicious of CRBSI as well as the companion blood cultures carried out to confirm the CRBSI diagnosis according to the criteria of Mermel et al. (2009) were retrospectively analyzed. Out of the 29 CVCs, 24 were actually associated with CRBSI and five were not (Table 3).

The microbial counts of the CVCs removed for suspicion of CRBSI as determined by BTA ranged from $7.6 * 10^{6}$ to $<10^{2}$ PE-CFUs and induced the change of BTA reagents in 3-18 h (Table 3). In particular, 18 CVCs induced the color change of BTA reagents within $8 \mathrm{~h}$ (microbial load ranging from $3.2 * 10^{7}$ to $2.3 * 10^{4} \mathrm{PE}-\mathrm{CFUs}$ ) and all were 
Table 3

Correlation between the performance of BioTimer Assay in counting microorganisms colonizing central venous catheters and catheter-related bloodstream infection.

\begin{tabular}{|c|c|c|c|c|}
\hline \multirow{2}{*}{$\begin{array}{l}\mathrm{CVCs}^{\mathrm{a}} \\
\text { (no.) }\end{array}$} & \multicolumn{2}{|l|}{ BioTimer Assay } & \multicolumn{2}{|l|}{ CRBSI $^{\mathrm{d}}$} \\
\hline & $\begin{array}{l}\text { Changing time }{ }^{\mathrm{b}} \\
\text { (hours) }\end{array}$ & $\begin{array}{l}\text { PE-CFUs/CVC } \\
\text { (range) }\end{array}$ & Confirmed & Non confirmed \\
\hline 3 & $\leq 4$ & $3.2 * 10^{7}-2.4 * 10^{6}$ & 3 & 0 \\
\hline 5 & $5-6$ & $2.2 * 10^{6}-1.3 * 10^{5}$ & 5 & 0 \\
\hline 10 & $7-8$ & $7.5 * 10^{5}-2.3 * 10^{4}$ & 10 & 0 \\
\hline 9 & $11-12$ & $3.4 * 10^{3}-2.2 * 10^{2}$ & 6 & 3 \\
\hline 2 & $\geq 12$ & $1.2 * 10^{3}-<10^{2}$ & 0 & 2 \\
\hline \multicolumn{5}{|l|}{ Total } \\
\hline 29 & & & 24 & 5 \\
\hline
\end{tabular}

a CVCs: central venous catheter.

${ }^{\mathrm{b}}$ Changing time of BioTimer Assay reagent(s).

${ }^{\mathrm{c}}$ Microbial counts were calculated using $S$. epidermidis and $P$. aeruginosa correlation lines and expressed as PE-CFUs (See Section 2.2 for details).

${ }^{\mathrm{d}}$ CRBSI: catheter-related bloodstream infection.

associated with CRBSI. Among the nine CVCs inducing the change of BTA reagents between 11 and $12 \mathrm{~h}$ (microbial load ranging from $3.4 * 10^{3}$ to $2.2 * 10^{2}$ PE-CFUs), six were associated with CRBSIs (66.6\% of CVCs) and three were not (33.3\% of CVCs). Finally, the two CVCs that induced the change of BTA reagent in $>12 \mathrm{~h}$ (microbial load of $1.3 * 10^{3}$ and $<10^{2}$ PE-CFUs) were not associated with CRBSI. Based on these observations, we evaluated the possibility of considering BTA as a rapid alert of CRBSIs. In this respect, the $12 \mathrm{~h}$-time was considered as the cut-off to discriminate between the possible and non-possible association of the changing time of BTA reagents and CRBSI. Therefore, we calculated that with respect to the diagnosis of CRBSI, BTA showed the sensitivity equal to $100 \%$, specificity of $40.0 \%$, positive and negative predictive values equal to 88.8 and $100 \%$, respectively. Taking together these data, we had strong evidence that this novel biological method can actually represent an early alert of CRBSI.

\section{Discussion}

Previously, BTA was proven reliable to count microbial biofilm adherent to abiotic surfaces (Pantanella et al., 2008; De Giusti et al., 2011; Berlutti et al., 2014). Of note, BTA is performed without sample manipulation thus overcoming the bias related to CVC intra-luminal colonization and to vortex or sonication procedures (Freitas et al., 2014; Pantanella et al., 2013; Srivastava and Bhargava, 2016). In particular, vortex procedures do not guarantee the detachment of all microbes as confirmed by the data reported here (Fig. 2). As matter of fact, BTA revealed residual adherent $S$. epidermidis and $P$. aeruginosa after vortexing. Of note, the counts of residual adherent bacteria were similar or higher than those of detached ones. This observation may be of interest since the microbial count is a critical parameter in recognizing CVC colonization.

BTA was applied to analyze the CVCs removed from hospitalized patients in comparison to RMM. In particular, BTA allowed: i) the determination of the sterility of CVCs; ii) to count bacteria adherent to CVCs; iii) to assess the presence of fermenting or non-fermenting microbes; iv) to have an early response.

Firstly, BTA correctly recognized as sterile or non-sterile all CVCs since no false positive and negative result was recorded $(100 \%$ concordance with RMM). The count of the adherent microbial population is of paramount importance, as this quantitative criterion coupled with microbial identification allowed the recognition of the colonized CVCs (Mermel et al., 2009). It should be underlined that BTA was performed after RMM that detached microorganisms from CVCs. If only BTA is performed, it seems reasonable to expect higher microbial counts than that obtained. In any case, applying the criteria of RMM (i.e.: microbial count and identification), BTA correctly recognized all colonized CVCs
(100\% agreement with RMM).

As noted above, BTA differentiated between fermenting and nonfermenting bacteria (Table 1). In this respect, BTA gave correct information in 34 out 35 CVCs (97.1\% agreement). The sole discrepancy concerned CG CVC that was colonized by both non-fermenting and fermenting bacteria as determined by RMM while BTA analysis indicated the presence of non-fermenting bacteria. This discrepancy may be due to the different proportion of non-fermenting and fermenting bacteria being the first the most abundant. It should be recalled that Candida spp. changed BTA reagents similarly to fermenting bacteria. Even if the BTA reagents were not devoted to counting Candida spp., this result strongly suggests that BTA-PR reagent can be applied to count Candida spp. thus hypothesizing a further application of BTA.

An additional relevant performance of BTA was the promptness of response. Indeed, the time needed to recognize sterile CVCs was shortened to about 2.0 -fold in comparison to RMM ( $24 \mathrm{vs} 48 \mathrm{~h}$ ). To evaluate if the $24 \mathrm{~h}$-time of incubation was actually reliable to assess CVC sterility, the non-changed BTA reagents were further incubated. The color of BTA reagents did not change after $48 \mathrm{~h}$ of incubation thus confirming that $24 \mathrm{~h}$ of incubation was reliable in determining CVC sterility. Therefore, the $24 \mathrm{~h}$-time could be considered also the cut-off time to discriminate between sterile and non-sterile CVCs. As a consequence, using BTA the bacterial counts of non-sterile CVCs were obtained within $24 \mathrm{~h}$ thus shortening the time to recognize non-sterile CVCs to about 3-fold (24 vs $72 \mathrm{~h}$ ).

Finally, a peculiar characteristic of BTA performance was related to the correlation between the changing time of BTA reagents and CRBSI. Bloodstream infections must be diagnosed in a timely manner so that adequate antimicrobial therapy can be started (Watson and Al-Hasan, 2014; Buehler et al., 2016). Unfortunately, the blood culture is timeconsuming due to the necessity of microbial growth while BTA does not require bacterial growth since it is based on the measure of microbial metabolism. Interestingly, $100 \%$ and $67 \%$ of the CVCs inducing the change of BTA reagent(s) within 8 and $12 \mathrm{~h}$, respectively, corresponded to CRBSI diagnosis (Table 3). Since the time of BTA response is inversely related to microbial load, a rapid change of BTA reagents indicates a high number of colonizing bacteria on CVCs thus alerting the physician to the possibility of CRBSI. Considering the cut-off of $12 \mathrm{~h}$, the high values of sensitivity, positive, and negative predictive values strongly suggest a possible use for BTA as an early alert of CRBSIs.

In conclusion, even if a limited number of CVCs was examined, the data are encouraging since BTA can give reliable indications in a very short time. In our opinion, BTA and the related laboratory procedure should be incorporated into routine microbiological evaluations of CVC colonization to improve the performance of routine microbiological methods.

\section{Funding}

This work was partially supported by the Regione Lazio, Italy to PV, Project: "Education and transfer of innovative methodologies", 2015-2017.

\section{References}

Berlutti, F., Rosso, F., Bosso, P., Giansanti, F., Ajello, M., De Rosa, A., Farina, E., Antonini, G., Valenti, P., 2003. Quantitative evaluation of bacteria adherent to polyelectrolyte HEMA-based hydrogels. J. Biomed. Mater. Res. A 67 (1), 18-25.

Berlutti, F., Frioni, A., Natalizi, T., Pantanella, F., Valenti, P., 2014. Influence of subinhibitory antibiotics and flow condition on Staphylococcus aureus ATCC 6538 biofilm development and biofilm growth rate: BioTimer Assay as a study model. J Antibiot. 67 (11), 763-769.

Buehler, S.S., Madison, B., Snyder, S.R., Derzon, J.H., Cornish, N.E., Saubolle, M.A., Weissfeld, A.S., Weinstein, M.P., Liebow, E.B., Wolk, D.M., 2016. Effectiveness of practices to increase timeliness of providing targeted therapy for inpatients with bloodstream infections: a laboratory medicine best practices systematic review and meta-analysis. J. Clin. Microbiol. 29 (1), 59-103.

Chopra, V., O'Horo, J.C., Rogers, M.A., Maki, D.G., Safdar, N., 2013. The risk of bloodstream infection associated with peripherally inserted central catheters compared 
with central venous catheters in adults: a systematic review and meta-analysis. Infect. Control Hosp. Epidemiol. 34 (9), 908-918.

Cleri, D.J., Corrado, M.L., Seligman, S.J., 1980. Quantitative culture of intravenous catheters and other intravascular inserts. J Infect Dis 141 (6), 781-786.

De Giusti, M., Berlutti, F., Pantanella, F., Marinelli, L., Frioni, A., Natalizi, T., Tufi, D., Valenti, P., 2011. A New Biosensor to Enumerate Bacteria in Planktonic and Biofilm Lifestyle. In: Serra, P.A. (Ed.), Biosensors-Emerging Materials and Applications. InTechOpen, Croatia, pp. 547-568.

Dobbins, B.M., Catton, J.A., Kite, P., McMahon, M.J., Wilcox, M.H., 2003. Each lumen is a potential source of central venous catheter-related bloodstream infection. Crit. Care Med. 31 (6), 1688-1690.

Donlan, R.M., Costerton, J.W., 2002. Biofilms: survival mechanisms of clinically relevant microorganisms. Clin Microbiol 15 (2), 167-193.

Freitas, A.I., Vasconcelos, C., Vilanova, M., Cerca, N., 2014. Optimization of an automatic counting system for the quantification of Staphylococcus epidermidis cells in biofilms. J. Basic Microbiol. 54 (7), 750-757.

Gahlot, R., Nigam, C., Kumar, V., Yadav, G., Anupurba, S., 2014. Catheter-related bloodstream infections. Int J Crit Illn Inj Sci 4 (2), 162-167.

Gominet, M., Compain, F., Beloin, C., Lebeaux, D., 2017. Central venous catheters and biofilms: where do we stand in 2017? APMIS 125 (4), 365-375.

Guembe, M., Martín-Rabadán, P., Cruces, R., Pérez Granda, M.J., Bouza, E., 2016. Rollplate alone does not demonstrate colonization in silicone neonatal catheters. Pediatr. Infect. Dis. J. 35 (3), 351-353.

Hall-Stoodley, L., Costerton, J.W., Stoodley, P., 2004. Bacterial biofilms: from the Natural environment to infectious diseases. Nat. Rev. Microbiol. 2 (2), 95-108.

Hess, D.J., Henry-Stanley, M.J., Wells, C.L., 2011. Gentamicin promotes Staphylococcus aureus biofilms on silk suture. J. Surg. Res. 170 (2), 302-308.

Kaye, K.S., Marchaim, D., Chen, T.Y., Baures, T., Anderson, D.J., Choi, Y., Sloane, R., Schmader, K.E., 2014. Effect of nosocomial bloodstream infections on mortality, length of stay, and hospital costs in older adults. J. Am. Geriatr. Soc. 62 (2), 306-311.

Maki, D.G., Weise, C.E., Sarafin, H.W.A., 1977. semiquantitative culture method for identifying intravenous-catheter-related infection. N. Engl. J. Med. 296 (23), 1305-1309.

Mermel, L.A., Allon, M., Bouza, E., Craven, D.E., Flynn, P., O'Grady, N.P., Raad, I.I., Rijnders, B.J., Sherertz, R.J., Warren, D.K., 2009. Guideline for the Diagnosis and Management of Intravascular Catheter-Related Infection: 2009 Update by the Infectious Diseases Society of America. Clin. Infect. Dis. 49 (1), 1-45.
Pantanella, F., Valenti, P., Frioni, A., Natalizi, T., Coltella, L., Berlutti, F., 2008. BioTimer Assay, a new method for counting Staphylococcus spp. in biofilm without sample manipulation applied to evaluate antibiotic susceptibility of biofilm. J. Microbiol. Methods 75 (3), 478-484.

Pantanella, F., Berlutti, F., Passeri, D., Sordi, D., Frioni, A., Natalizi, T., Terranova, M.L., Rossi, M., Valenti, P., 2011. Quantitative evaluation of bacteria adherent and in biofilm on single wall carbon nano-tube coated surface. Interdiscip Perspect Infect Dis 2011, 1-9.

Pantanella, F., Valenti, P., Natalizi, T., Passeri, D., Berlutti, F., 2013. Analytical techniques to study microbial biofilm on abiotic surfaces: pros and cons of the main techniques currently in use. Ann. Ig. 25 (1), 31-42.

Pratt, R.J., Pellowe, C., Loveday, H.P., Robinson, N., Smith, G.W., Barrett, S., Davey, P., Harper, P., Loveday, C., McDougall, C., Mulhall, A., Privett, S., Smales, C., Taylor, L., Weller, B., Wilcox, M., 2001. The epic project: developing national evidence-based guidelines for preventing healthcare associated infections. Phase I: guidelines for preventing hospital-acquired infections. Department of Health (England). J. Hosp. Infect. 47, S3-S82.

Romeo, U., Palaia, G., Nardo, A., Tenore, G., Telesca, V., Kornblit, R., Del Vecchio, A. Frioni, A., Valenti, P., Berlutti, F., 2015. Effectiveness of KTP laser versus $980 \mathrm{~nm}$ diode laser to kill Enterococcus faecalis in biofilms developed in experimentally infected root canals. Aust. Endod. J. 41 (1), 17-23.

Sherertz, R.J., Raad, I.I., Balani, A., Koo, L.C., Rand, K.H., Pickett, D.L., Straub, S.A., Fauerbach, L.L., 1990. Three-year experience with sonicated vascular catheter cultures in a clinical microbiology laboratory. J. Clin. Microbiol. 28 (1), 76-82.

Srivastava, S., Bhargava, A., 2016. Biofilms and human health. Biotechnol. Lett. 38 (1), $1-22$.

Watson, C.M., Al-Hasan, M.N., 2014. Bloodstream infections and central line-associated bloodstream infections. Surg. Clin. North Am. 94 (6), 1233-1244.

Wells, C.L., Henry-Stanley, M.J., Barnes, A.M., Dunny, G.M., Hess, D.J., 2011. Relation between antibiotic susceptibility and ultrastructure of Staphylococcus aureus biofilms on surgical suture. Surg. Infect. 12 (4), 297-305.

Wengrovitz, M., Spangler, S., Martin, L.F., 1991. Sonication provides maximal recovery of Staphylococcus epidermidis from slime-coated vascular prosthetics. Am. Surg. 57 (3), 161-164.

Yousif, A., Jamal, M.A., Raad, I., 2015. Biofilm-based central line-associated bloodstream infections. Adv. Exp. Med. Biol. 830, 157-179. 